\title{
Das Zusammenspiel individuellen und organisationalen Lernens - Ein Review der neueren Literatur
}

\author{
Felix Mynarek ${ }^{1,2} \cdot$ Jennifer Steckel ${ }^{3} \cdot$ Axel Grandpierre $^{2} \cdot$ Karin Häring $^{2}$
}

Angenommen: 23. September 2021 / Online publiziert: 27. Oktober 2021

(c) Der/die Autor(en) 2021

\section{Zusammenfassung}

Die Bedeutung des Lernens in und von Organisationen verdeutlicht sich in der hohen Anzahl wissenschaftlicher Publikationen und ist ebenso in den Bemühungen von Organisationen, organisationales Lernen in den Fokus zu nehmen, erkennbar. Organisationales Lernen soll dazu beitragen, sich nachhaltig verbessern zu können, agiler und resilienter zu werden. Das Literatur-Review zielt darauf ab, die Bedeutung des Lernens im Zeitraum 2016-2021 mithilfe der Datenbanken ScienceDirect, Sage Journals und Wiley von einer individuellen bis hin zu einer organisationalen Ebene zu untersuchen und hier insbesondere Faktoren zu identifizieren, die das Zusammenspiel der drei Akteursebenen (Individuum, Gruppe, Organisation) beeinflussen und damit organisationales Lernen bedingen. Das Literatur-Review zeigt auf, dass Lernen in Organisationen zum einen auf dem Zusammenspiel der genannten Ebenen gedacht werden muss und zum anderen sowohl individuelle, gruppenbasierte als auch organisationale Faktoren einen Einfluss auf das Lernen haben. Die Ergebnisse lassen insbesondere den Schluss zu, dass die Interaktion in der Organisation und dabei der Wissensaustausch bzw. das Teilen von Wissen zu den relevanten Einflussfaktoren organisationalen Lernens gehören.

Praktische Relevanz: Organisationen sehen sich mit einem dynamischen Umfeld und komplexen Fragestellungen konfrontiert. Gesellschaftliche, wirtschaftliche und technologische Neuerungen mit teils disruptivem Charakter erfordern von ihnen, ihre Fähigkeit zur Resilienz zu verstärken und organisationales Lernen strategisch zu nutzen.

Schlüsselwörter Organisationales Lernen · Individuelles Lernen · Teamlernen · Wissensaustausch · Literatur-Review

\section{The interplay of individual and organizational learning-A review of recent literature}

\section{Abstract}

The importance of learning in and by organizations is reflected in the high number of scientific publications and is also evident in the efforts of organizations to focus on organizational learning. Organizational learning should contribute to sustainable improvement, agility and resilience. The literature review aims to investigate the significance of learning in the period 2016-2021 with the help of the databases ScienceDirect, Sage Journals and Wiley from an individual to an organizational level and to identify in particular factors that influence the interaction of the three actor levels (individual, group, organization) and thus condition organizational learning. The literature review shows that learning in organizations has to be thought on the interaction of the mentioned levels on the one hand and that individual, group-based as well as organizational factors have an influence on learning on the other hand. In particular, the results allow the conclusion that interaction in the organization and, in this context, knowledge sharing are among the relevant factors influencing organizational learning.

Felix Mynarek, M.A.

felix.mynarek@t-online.de

1 Institut für Marketing \& Management (570B), Universität Hohenheim, Schwerzstr. 42, 70599 Stuttgart, Deutschland
2 Europäische Fachhochschule Rhein/Erft GmbH, Kaiserstraße 6, 50321 Brühl, Deutschland

3 Bergische Universität Wuppertal, Gaußstraße 20, 42119 Wuppertal, Deutschland 
Practical Relevance: Organizations are confronted with a dynamic environment and complex issues. Social, economic and technological changes, partly of a disruptive nature, require organizations to strengthen their capacity for resilience and to make strategic use of organizational learning.

Keywords Organizational learning $\cdot$ Individual learning $\cdot$ Team learning $\cdot$ Knowledge sharing $\cdot$ Literature review

\section{Einleitung}

Die Fähigkeit einer Organisation zu lernen, stellt eine relevante Quelle dar, um sich kontinuierlich zu verbessern und gegenüber gesellschaftlichen, wirtschaftlichen und technologischen Neuerungen mit teils disruptivem Charakter resilient zu werden. Individuen stellen dabei die kleinste Lerneinheit von Organisationen dar, weshalb organisationales Lernen, über einen langen Zeitraum der Forschungshistorie hinweg, als vom individuellen Lernen vollständig abhängiges System betrachtet wurde (Wang und Ahmed 2002). So wurde das Individuum als agent für das Lernen von Organisationen aufgefasst (Argyris und Schön 1978). Dieser Vorstellung nach findet organisationales Lernen dann statt, wenn Individuen innerhalb einer Organisation eine problematische Situation erfahren und sich für die Organisation mit diesem Problem auseinandersetzen. Diese Auseinandersetzung führt dann bei dem Individuum zu einer Umstrukturierung von Handlungsmustern und Aktivitäten, welche die organisationalen Strukturen verändert (Argyris und Schön 1996, S. 16). Betrachtet man sozial kognitive Lerntheorien, die Lernen als Zusammenspiel sozialer und kognitiver Prozesse auffassen, so gewinnt kollektives Lernen, d.h. das Lernen in Gruppen, für organisationales Lernen an Bedeutung (Wang und Ahmed 2002). Organisationales Lernen muss demzufolge von der kleinsten Lerneinheit, dem Individuum, über das Lernen in Gruppen hin zur Organisationsebene gedacht werden, wobei der Wille des Individuums, zu lernen, den Ausgangspunkt organisationalen Lernens darstellt. Schon Crossan et al. (1999) und auch neuere Arbeiten von Akinci und Sadler-Smith (2019) nehmen das Zusammenspiel des Lernens auf den drei Ebenen (Individuum, Gruppe und Organisation) in den Blick und beschreiben die dabei ablaufenden Prozesse. Wenn organisationales Lernen eine zentrale Voraussetzung für die Anpassung und Weiterentwicklung von Strukturen und Verhaltensweisen innerhalb der Organisation darstellt, so ergibt sich die Frage welche Faktoren individuelles Lernen auf der Gruppenebene sowie auf der organisationale Ebene transformiert. Aufgrund der sich schnell ändernden Rahmenbedingungen organisationaler Strukturen, z. B. durch den digitalen Wandel, zielt das vorliegende Literatur-Review darauf ab, die Bedeutung des Lernens von einer individuellen bis hin zu einer organisationalen Ebene zu untersuchen und hier insbesondere Faktoren zu identifizieren, die das
Zusammenspiel dieser drei Ebenen beeinflussen und damit organisationales Lernen bedingen.

Der vorliegende Beitrag soll zunächst ein einheitliches Verständnis über das Lernen, ausgehend von einer individuellen, den zugehörigen lerntheoretischen Ansätzen, bis hin zu einer organisationalen Ebene liefern. Danach werden die Fragestellung und die Methodik des Literatur-Reviews beschrieben. Im fünften Kapitel werden die Ergebnisse mit Schwerpunkten zwischen den Dimensionen individuelles Lernen mit organisationalen Lernen sowie kollektives Lernen benannt und in dem darauffolgenden Kapitel diskutiert.

\section{Organisationales Lernen}

Organizations are getting busier, but can they still learn to get better? (Desai 2020)

Lernen ist der entscheidende Faktor für den Erfolg von Organisationen (Cerasoli et al. 2018) und wird als soziale Dimension betrachtet, wodurch aktive Lernprozesse entstehen (Furlan et al. 2019). Lernen entsteht durch Erfahrungen, die eine Veränderung im Wissen hervorrufen (Levitt und March 1988). Organisationales Lernen ist dabei kein fest definierter Begriff, sondern kann als multiperspektivisches Konstrukt verstanden werden (Aponte und Zapata 2013). Der vorliegenden Arbeit liegt die behaviorale Perspektive organisationalen Lernens zugrunde, die davon ausgeht, dass Lernen sowohl kognitive als auch behaviorale Veränderungen mit sich bringt (Vera et al. 2012). Organisationales Lernen bedeutet hier, dass Organisationen ihre Routinen und ihr Verhalten abhängig von ihren Zielen ändern (Greve et al. 2019, S. 1759; Levitt und March 1988; Argote und Levine 2020; Greco et al. 2019). Der Begriff des organisationalen Lernens ist seit über 50 Jahren präsent und hat zunehmend an Bedeutung gewonnen (Crossan et al. 1999; Cerasoli et al. 2018). Organisationales Lernen wird als Prozess verstanden, der die Veränderung der Kognition und des Verhaltens bei Individuen sowie des gemeinsamen Denkens und Handelns impliziert (Vera et al. 2012). Das Lernen in und von Organisationen wird als wichtigstes Instrument in Umstrukturierungsprozessen in Organisationen angesehen (Crossan et al. 1999) und soll die Überlebensfähigkeit eines Unternehmens als auch dessen Wettbewerbsvorteile sichern (Greco et al. 2019). Annosi et al. (2018) betonen, dass organisationales Lernen dann stattfindet, wenn Organisationen 
neues Wissen nutzen, um ihre bestehenden Abläufe anzupassen.

In ihrem organisational learning framework gehen Crossan et al. (1999, S. 523) von vier zentralen Annahmen organisationalen Lernens aus:

1. Organisationales Lernen umfasst das Spannungsfeld zwischen der Assimilation neuen Wissens (Exploration) und der Nutzung des bereits Gelernten (Exploitation).

2. Organisationales Lernen erfolgt auf mehreren Ebenen: individuelle, kollektive und organisationale Ebene.

3. Die drei Ebenen des organisationalen Lernens sind über soziale und psychologische Prozesse miteinander verbunden: Intuition, Interpretation, Integration und Institutionalisierung.

4. Die Kognition beeinflusst das Verhalten; das Verhalten beeinflusst die Kognition.

Das von Crossan et al. (1999) entwickelte Modell organisationalen Lernens (4 I framework of organizational learning) umfasst vier zentrale miteinander zusammenhängende Prozesse, die auf den drei Ebenen des Lernens stattfinden. Während der Prozess der Intuition eindeutig der individuellen Ebene und der Prozess der Institutionalisierung der organisationalen Ebene zugeordnet werden können, fungieren die Prozesse der Interpretation und der Integration als Überbrückungsprozesse zwischen den Ebenen (Abb. 1).

Organisationales Lernen wird in diesem Modell als dynamischer Prozess verstanden und über Feed-forward- und Feedback-Prozesse gesteuert. Über Feed-forward Prozesse, die sich auf den Erwerb und die Konstruktion neuen Wissens beziehen, wird Lernen von der individuellen über die Gruppenebene hin zur Organisationsebene betrachtet. Im Vergleich hierzu wird unter Feedback-Prozessen die Nutzung bereits vorhandenen Wissens von der Organisationsebene über die Gruppenebene hin zur individuellen Ebene verstanden (Crossan et al. 1999). Organisationales Lernen erfolgt nach Crossan et al. (1999) dann, wenn individuelles und kollektives Lernen institutionalisiert werden. Auch Aponte und Zapata (2013) definieren organisationales Lernen als Prozess der Denk- und Verhaltensänderung auf Seiten der Individuen wie auch als Prozess der Institutionalisierung von Wissen. Die Qualität individuellen wie auch kollektiven Lernens stellen somit Schlüsseldeterminanten des organisationalen Lernerfolgs dar (Hayes und Allinson 1998, S. 847). Mit Berücksichtigung des 4 I framework of organizational learning von Crossan et al. (1999) kann Lernen im Sinne der Intuition auf der rein individuellen Ebene, andererseits aber auch im Sinne der Interpretation als sozialer Prozess verstanden werden.

Akinci und Sadler-Smith (2019) gehen in ihrem Beitrag von der folgenden Annahme aus: „Organizational learning occurs as a result of cognitions initiated by individuals' intuitions transcending to the group level by way of articulations and interactions between the group members through the processes of interpreting (cf. ,externalization ") and integrating, and which become institutionalized at the organization level“" (Akinci und Sadler-Smith 2019, S. 560). An dieser Stelle zeigt sich eine Anpassung des Modells von Crossan et al. (1999). Crossan et al. (1999) nehmen in ihrem Modell einen direkten Zusammenhang zwischen dem Lernen auf der Ebene des Individuums und der Ebene der Organisation an. Im Vergleich hierzu gehen Akinci und Sadler-Smith (2019; Abb. 2) davon aus, dass individuelles Lernen nicht unmittelbar im Lernen auf der Ebene der Organisation mündet. Hier betrachten sie vor allem das Lernen auf Gruppenebene als Bindeglied zwischen dem Lernen auf der Ebene des Individuums und der Ebene der Organisation.

Die auf individueller Ebene konstruierten Intuitionen werden durch Kommunikationsprozesse interpretiert und so zu kollektiven Intuitionen transformiert. Die kollektiven
Abb. 1 Organisatorisches Lernen als dynamischer Prozess. (Crossan et al. 1999, S. 532)

Fig. 1 Organizational Learning As a Dynamic Process. (Crossan et al. 1999, p. 532)

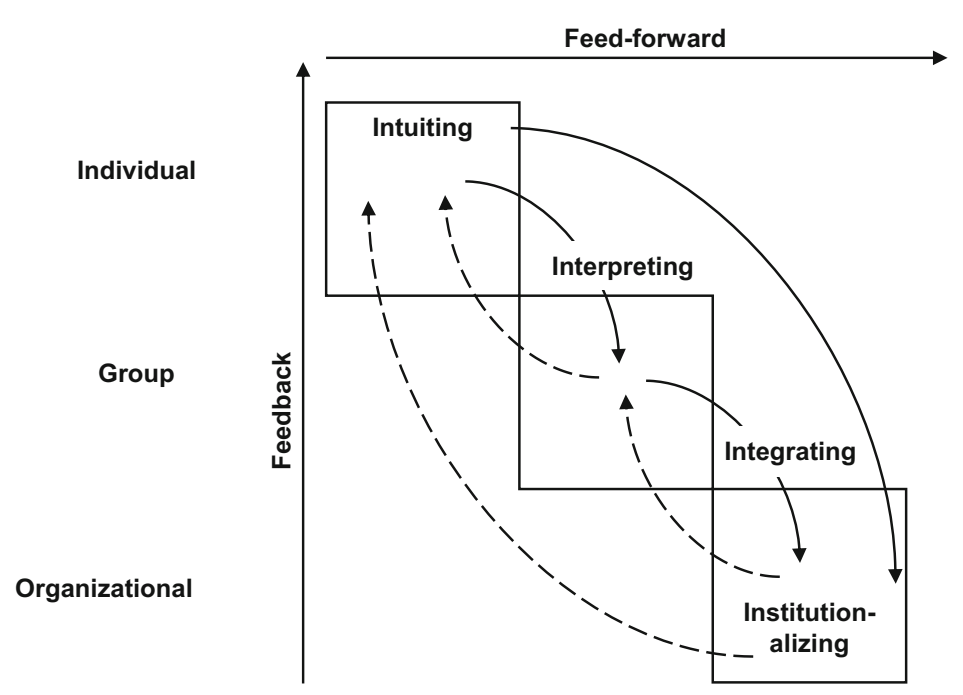


Abb. 2 Feed-forward- und Feedback-Schleifenprozesse des organisatorischen Lernens. (Akinci und Sadler-Smith 2019, S. 568)

Fig. 2 Feed-forward and feedback loop processes of organizational learning. (Akinci and Sadler-Smith 2019, p. 568)

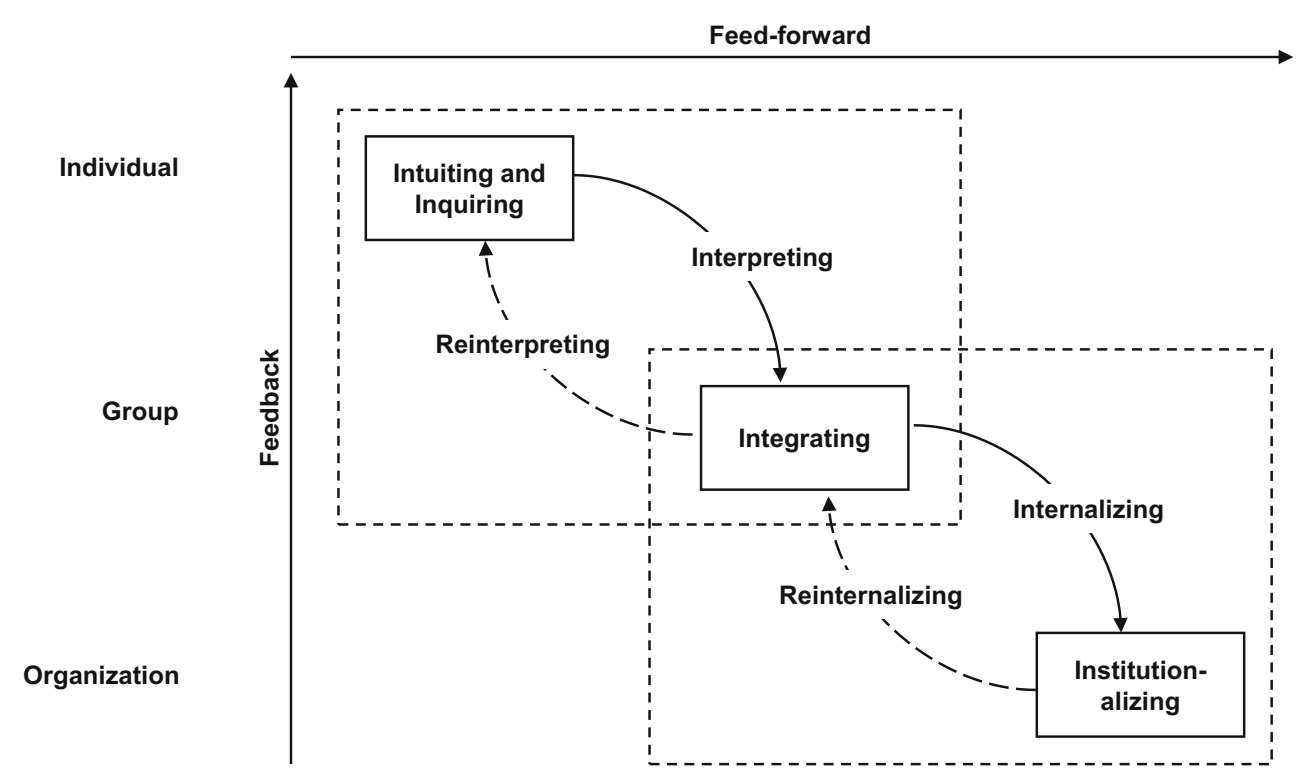

Intuitionen dienen dabei als Grundlage von Gruppenentscheidungen und sind durch den Prozess der Integration gekennzeichnet. Die Ebene der Organisation wird als ,,institutionalizing“ bezeichnet. Die Rückkopplungsschleifen zwischen den einzelnen Ebenen stehen für eine Neuinterpretation und Verinnerlichung von kollektiven Lerninhalten. Die Dimension der „Interpretation“ wird in der Rückkopplungsschleife manifestiert, die sich über die Ebenen der Organisation, des Individuums und der Gruppe verteilt und einen ,,interaktiven Prozess zwischen Kognition und Handlung" hervorruft (Akinci und Sadler-Smith 2019, S. 571).

\subsection{Individuelles Lernen - Lerntheoretische Ansätze}

Lernen ist ein komplexer kognitiver Prozess, welcher emotionale und motivationale Ressourcen berücksichtigt und sich klassisch am einzelnen Lernenden orientiert (Cerasoli et al. 2018). Konstruktivistische Lerntheorien gehen davon aus, dass Lernen ein aktiver Prozess der Wissenskonstruktion ist (Adams 2006), der auf Erfahrungen des Individuums basiert (McLeod 2003). Die Relevanz von Erfahrungen betont auch Lefrancois et al. (1986, S. 3), demgemäß „Lernen [...] alle Verhaltensänderungen [umfasst], die aufgrund von Erfahrungen zustande kommen“. Der Prozess der Wissenskonstruktion kann als Transformation von Erfahrungen beschrieben werden (Kolb 1984). Dieser Prozess wird im Modell von Crossan et al. (1999, S. 525) als Prozess der Intuition beschrieben und als ,preconscious recognition of the pattern and/or possibilities inherent in a personal stream of experience" verstanden. Intuition wird primär über fehlendes Bewusstsein charakterisiert (Hogarth 2001) und markiert gleichzeitig den Beginn eines neuen Lernprozesses (Crossan et al. 1999). In Anlehnung an die sozial-kon- struktivistische Perspektive des Lernens kann angenommen werden, dass organisationales Lernen bzw. organisationale Transformationsprozesse auf bewussten Erfahrungen basieren (Bandura 1988). Um somit individuell konstruiertes Wissen für organisationale Transformationsprozesse nützlich zu machen, bedarf es daher zunächst des Bewusstseins über diese Erfahrungen (Aponte und Zapata 2013). Hier$\mathrm{zu}$ ist insbesondere der Prozess der Integration von Wissen von Bedeutung (Chonko et al. 2003), bei dem individuelles Wissen und individuelle Erfahrungen zwischen Individuen geteilt werden und so auf Gruppenebene integriert werden. Um individuelles Wissen und Erfahrungen auf Gruppenebene zu integrieren, bedarf es der Interpretation dieser. Die Interpretation erfolgt dabei sowohl auf individueller als auch auf kollektiver Ebene über Kommunikationsprozesse und wird von Crossan et al. (1999, S. 525) als ,the explaining through words and/or actions, of an insight or idea to one's self and to others" verstanden. Die Interpretation von Gefühlen oder Empfindungen findet individuell statt und eröffnet neben der Intuition inneren Prozess (Crossan et al. 1999). Lernen als Wissenskonstruktion und Transformation von Erfahrungen kann somit als Prozess sozialer Interaktion, Interpretation und Verstehen verstanden werden (Vygotsky 1975), bei dem die aktive Wissenskonstruktion innerhalb einer sozialen Gruppe durch die einzelnen Mitglieder sowie durch Austausch zwischen den Mitgliedern einer sozialen Gruppe kollektiv erfolgt.

\subsection{Individuelles Lernen und Organisationales Lernen}

Akinci und Sadler-Smith (2019) fokussieren in ihrem Modell insbesondere die bottom-up Perspektive organisationa- 
len Lernens, bei der Innovation und Wissenskonstruktion vom Individuum her gedacht wird. Gemäß des erfahrungsorientierten Ansatzes organisationalen Lernens, sind für den Entscheidungsfindungsprozess in Organisationen insbesondere Erfahrungen von besonderer Bedeutung (Klimecki und Thomae 1997). So werden individuelle Erfahrungen durch den Prozess der Sozialisierung zu geteilten Erfahrungen und bilden die Grundlage für organisationalen Lernens (Akinci und Sadler-Smith 2019). Entscheidungen werden dabei von Yates und Tschirhart (2006) als ,,...] a commitment to a course of action that is intended to yield results that are satisfying for specified individuals" (S. 24) verstanden. Chan (2003) charakterisiert individuelles Lernen als Katalysator für einen strategischen Wandel durch die Einbeziehung individuellen Engagements und individueller Fähigkeiten. Organisationales Lernen kann dabei über die Erstellung, den Erwerb und die Kommunikation von Wissen durch die einzelnen Mitglieder erfolgen (King 2001). Auch Kim (1993, S. 37) betont, dass jede Organisation aus Individuen besteht und Organisationen zwar unabhängig von bestimmten Individuen, nicht aber unabhängig von allen Individuen lernen können. Geng et al. (2018) heben die Bedeutung der Fähigkeiten und Kenntnisse von Mitarbeitenden für organisationales Lernen hervor, genauso wie die daraus entstehenden dynamischen Interaktionen. Nach Garratt (1990) tragen insbesondere drei zentrale individuelle Lernprozesse zum organisationalen Lernen bei: Gewinnung von Wissen, Teilen von Wissen und Nutzung von Wissen. Es ist allerdings anzumerken, dass individuelles Lernen nicht grundsätzlich und zwangsläufig zu organisationalem Lernen führt (Chan 2003). Das Wissen, welches Individuen konstruieren, liegt sowohl in implizierter als auch in expliziter Form vor. Implizites Wissen lässt sich dadurch charakterisieren, dass es dem Bewusstsein nur schwer zugänglich ist und somit schwierig zu versprachlichen ist. Im Vergleich hierzu ist explizites Wissen dem Lernenden zugänglich und kann somit leichter kommuniziert werden (Argote und Levine 2020). Für organisationale Lernprozesse sind demnach, nach Akinci und Sadler-Smith (2019) sowie nach Crossan et al. (1999), nur explizites relevant, da dieses kommuniziert und geteilt werden kann.

\subsection{Individuelles Lernen und kollektives Lernen}

Kollektives Lernen wird als Prozess definiert, bei dem individuell erworbenes Wissen in kollektiv geteiltes Wissen transformiert wird (Dechant et al. 2000). Organisationen können nur dann lernen, wenn es zwischen den Individuen innerhalb der Organisation kollektiv zu einem Erfahrungsund Wissensaustausch kommt (Berg 1993; Bennett und O’Brien 1994; Bierly und Hämäläinen 1995; Bain 1998; Brown und Duguid 1998). So sagt Senge (1990, S. 10), dass ,teams, not individuals, are the fundamental learning unit in organizations“. Studien belegen, dass das Teilen von Informationen und Wissen in einer kollektiven Leistung resultiert, die höher als die Leistung ist, die durch das einzelne Individuum erzielt wird (Mohrman et al. 1995; Sorrells-Jones 1999). Auch Chan (2003) konnte zeigen, dass kollektives Lernen signifikant mit organisationalem Lernen zusammenhängt. Hierbei wird von- und miteinander gelernt, indem unterschiedliche Annahmen besprochen werden (Mainert et al. 2018). Valentine (2017, S. 3) ergänzt, „dass Lernen von Natur aus örtlich begrenzt ist, was bedeutet, dass die betreffenden Aktivitäten in kleinen Gruppen am Ort der gemeinsamen Arbeit durchgeführt werden“. Kollektives Lernen wird hier als Bindeglied zwischen individuellem und organisationalem Lernen betrachtet Dechant et al. (2000).

\section{Fragestellung}

Organisationen können als soziales System (Endruweit 2004) ,wechselseitig abhängige[r] soziale[r] Verhaltensweisen einer Anzahl von Personen“ (March und Simon 1976, S. 81) verstanden werden. Lernen findet somit auf verschiedenen Ebenen statt: auf der individuellen, der kollektiven und der organisationalen Ebene. Organisationale Transformationsprozesse sind nach Gattermeyer und AlAni (2001) nur dann erfolgreich, wenn die jeweiligen Zielgruppen die Veränderungen zum einen ausreichend akzeptieren und zum anderen sich mit diesen Veränderungen identifizieren können. Für transformationale Prozesse ist es somit unabdingbar, Lernen auf den verschiedenen Akteursebenen als Zusammenspiel zu betrachten. Dies belegt auch der aktuelle Forschungsstand. Das vorliegende Literatur-Review geht über das Zusammenspiel des Lernens auf den verschiedenen Akteursebenen hinaus und zielt darauf ab, Faktoren zu identifizieren, die den Zusammenhang individuellen, kollektiven und organisationalen Lernens beeinflussen.

\section{Methode}

Zur Untersuchung der Fragestellung wurde ein systematisches Literatur-Review durchgeführt. Zunächst wurden für das Literatur-Review Suchkriterien festgelegt. Zum einen wurden nur Artikel berücksichtigt, die zwischen 2016 und 2021 veröffentlicht wurden sowie im englischoder deutschsprachigen Raum bereits einen Peer-Review durchlaufen haben. Als weiteres Suchkriterium wurden die folgenden Schlagwörter festgelegt: (1) individual learning AND organisational learning, (2) group learning AND organisational learning sowie (3) individual AND group learning. Die Schlagwörter sollten sich hierbei entweder im 
Titel oder im Abstract wiederfinden. Für die Durchführung der Suche wurden die Datenbanken: ScienceDirect, Sage Journals und Wiley verwendet. Insgesamt konnten 1209 Artikel gefunden werden, deren Abstracts in einem zweiten Schritt entsprechend folgender Einschlusskriterien analysiert wurden: (1) Organisationaler Kontext, (2) deutschoder englischsprachig, (3) Untersuchungsgegenstand: individuelles, kollektives oder organisationales Lernen. Die 126 in die Eignungsprüfung aufgenommenen Artikel wurden nun in einem letzten Schritt vollständig gesichtet und erneut mit Blick auf die obigen Einschlusskriterien sowie einem weiteren Einschlusskriterium (quantitatives Studiendesign) analysiert. Das Einschlusskriterium des quantitativen Studiendesigns lässt sich durch die dem Literatur-Review angelegten Fragestellung begründen, da Untersuchungen zum Einfluss von Faktoren quantitativen Daten notwendig machen. Schließlich wurden 16 Artikel in das LiteraturReview zur Beantwortung der Fragestellung aufgenommen.

\section{Ergebnisse}

Insgesamt konnten 16 Artikel herausgearbeitet werden, in denen Faktoren untersucht werden, die den Zusammenhang zwischen individuellem, kollektivem und organisationalem Lernen beeinflussen. Tab. 1 zeigt die 16 in das Literatur-Review einbezogenen Artikel sowie die Zusammenhänge der betrachteten Akteursebenen. Es wird insbesondere deutlich, dass für den Zeitraum 2016 bis 2021 zum Zusammenhang von kollektivem und organisationalem Lernen keine quantitativen Studien vorliegen.

Tab. 1 Überblick der Artikel und deren Untersuchungsgegenstand Table 1 Overview of the articles and their research subject

\begin{tabular}{lc}
\hline Autorenschaft & Jahr \\
\hline Individuelles und organisationales Lernen \\
Camps et al. & 2016 \\
Choi et al. & 2020 \\
Furlan et al. & 2019 \\
Li et al. & 2018 \\
Individuelles und kollektives Lernen & \\
Burmeister et al. & 2018 \\
Chan et al. & 2021 \\
Gerpott et al. & 2019 \\
Ghosh und Tripathi & 2020 \\
Han et al. & 2020 \\
Janardhanan et al. & 2020 \\
Kaše et al. & 2019 \\
Lee & 2019 \\
Lejarraga und Müller-Trede & 2017 \\
Rhee und Choi & 2017 \\
Zhang und Min & 2019 \\
Zhu et al. & 2018 \\
\hline
\end{tabular}

\subsection{Individuelles und organisationales Lernen}

Tab. 2 einbezogenen Artikel für die Betrachtung des $\mathrm{Zu}$ sammenhangs zwischen individuellem und organisationalem Lernen zusammenfassend dar. Camps et al. (2016) gehen in ihrer Studie der Frage nach, wie und in welchem Ausmaß die individuelle Leistung durch die organisationale Lernfähigkeit (OCL) und die Flexibilität der Mitarbeitenden in Umgebungen mit hoher Unsicherheit (Umgebungsschwankungen) verbessert werden. Der Studie liegt eine Fragebogenerhebung zugrunde, an der 174 Probandinnen und Probanden teilnahmen. Während die individuelle Leistung als Fremdeinschätzung erhoben wurde, wurde die organisationale Lernfähigkeit und die Flexibilität der Mitarbeitenden über Selbstberichtsbögen erfasst.

Weiterhin konnten Analysen einen signifikanten indirekten Einfluss organisationaler Lernfähigkeit auf die individuelle Leistung zeigen, was den mediierenden Einfluss der Flexibilität der Mitarbeitenden zeigt.

Studien belegen, dass das psychologische Kapital von Mitarbeitenden die Leistung des Individuums beeinflussen. Choi et al. (2020) greifen diese Befunde auf und gehen in ihrer Arbeit davon aus, dass der Einfluss des psychologischen Kapitals auf die individuelle Leistung durch die Beziehung zum informellen Lernen erklärt werden kann und über die Person-Umgebungs-Passung (PE) moderiert wird. Der Studie liegt eine Fragebogenerhebung zugrunde, an der 221 Probandinnen und Probanden teilnahmen.

Furlan et al. (2019) gehen in ihrer Studie davon aus, dass Individuen als Teil der Organisation nicht nur passiv lernen, sondern Lernen durch soziale Handlungen innerhalb der Organisation erfolgt. In ihrer Arbeit wird daher anhand des Problemlöseverhaltens der Einfluss von Lernmechanismen auf individueller wie auch auf organisationaler Ebene untersucht. Die Autorenschaft geht hier insbesondere auf die Wissensartikulation (OKA) und die Wissenskodierung (OKC) auf organisationaler Ebene, der wahrgenommenen Wissensartikulation (PKA) und der wahrgenommenen Wissenskodierung (PKC) auf individueller Ebene sowie deren Einfluss auf das Problemlöseverhalten ein. Der Studie liegt eine Fragebogenerhebung zugrunde, an der 383 Probandinnen und Probanden teilnahmen.

Li et al. (2018) basieren ihre Studie auf Forschungsbefunden, die belegen, dass das Wissensmanagement in komplexen Produktionssystemen nicht auf das Teilen expliziten Wissens reduziert werden darf, sondern ebenso betrachtet werden muss, wie implizites individuelles Wissen zwischen Gruppen oder zwischen Organisationen geteilt und in explizites Wissens überführt werden kann. Aus diesem Anlass gehen Li et al. (2018) dieser Frage nach und untersuchen Wissenskonstruktionsprozesse und deren Zusammenhang mit innovativen Leistungen innerhalb komplexer Produktionssysteme. Die Autorenschaft betrachtet hier insbesonde- 


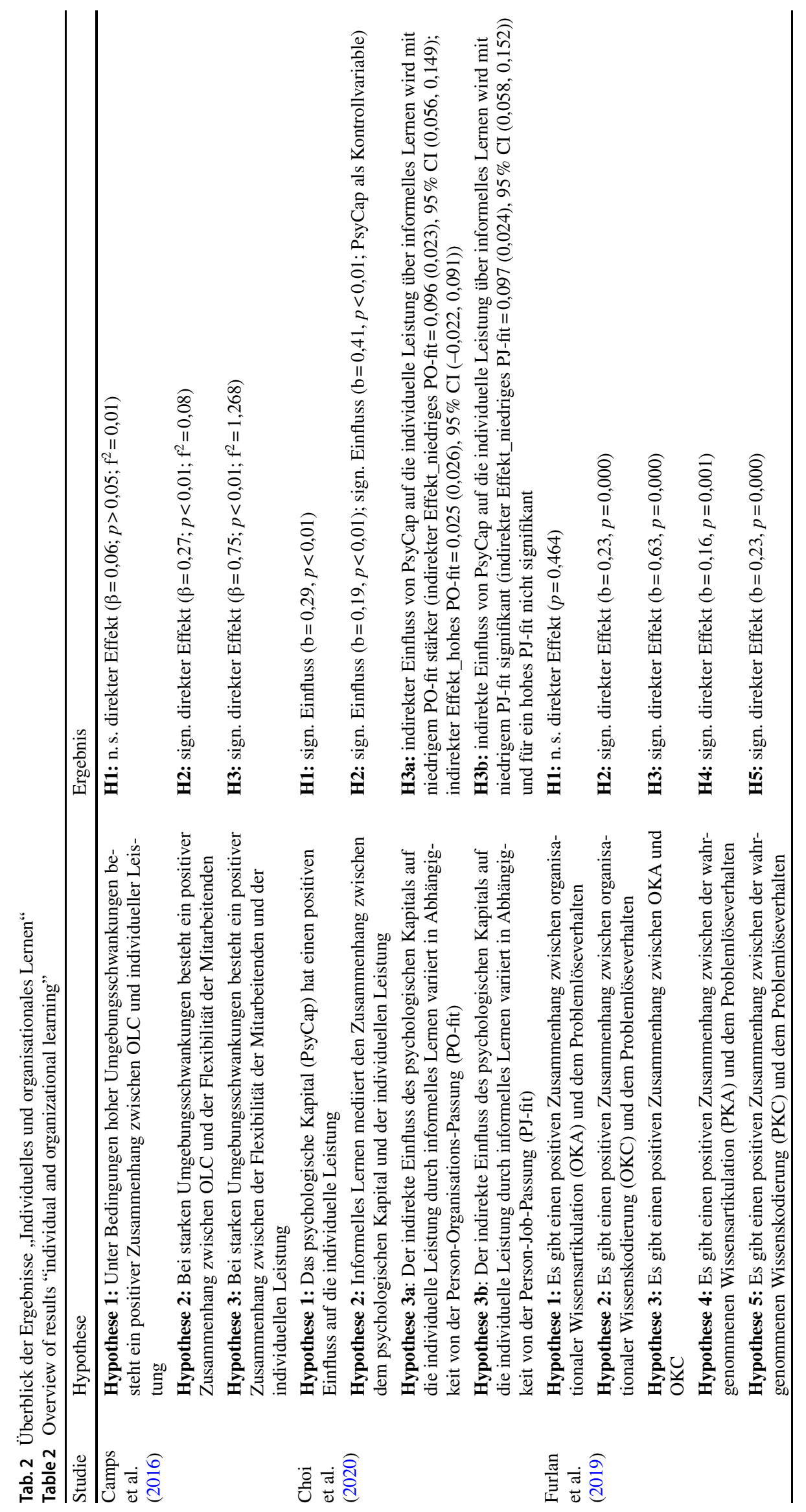




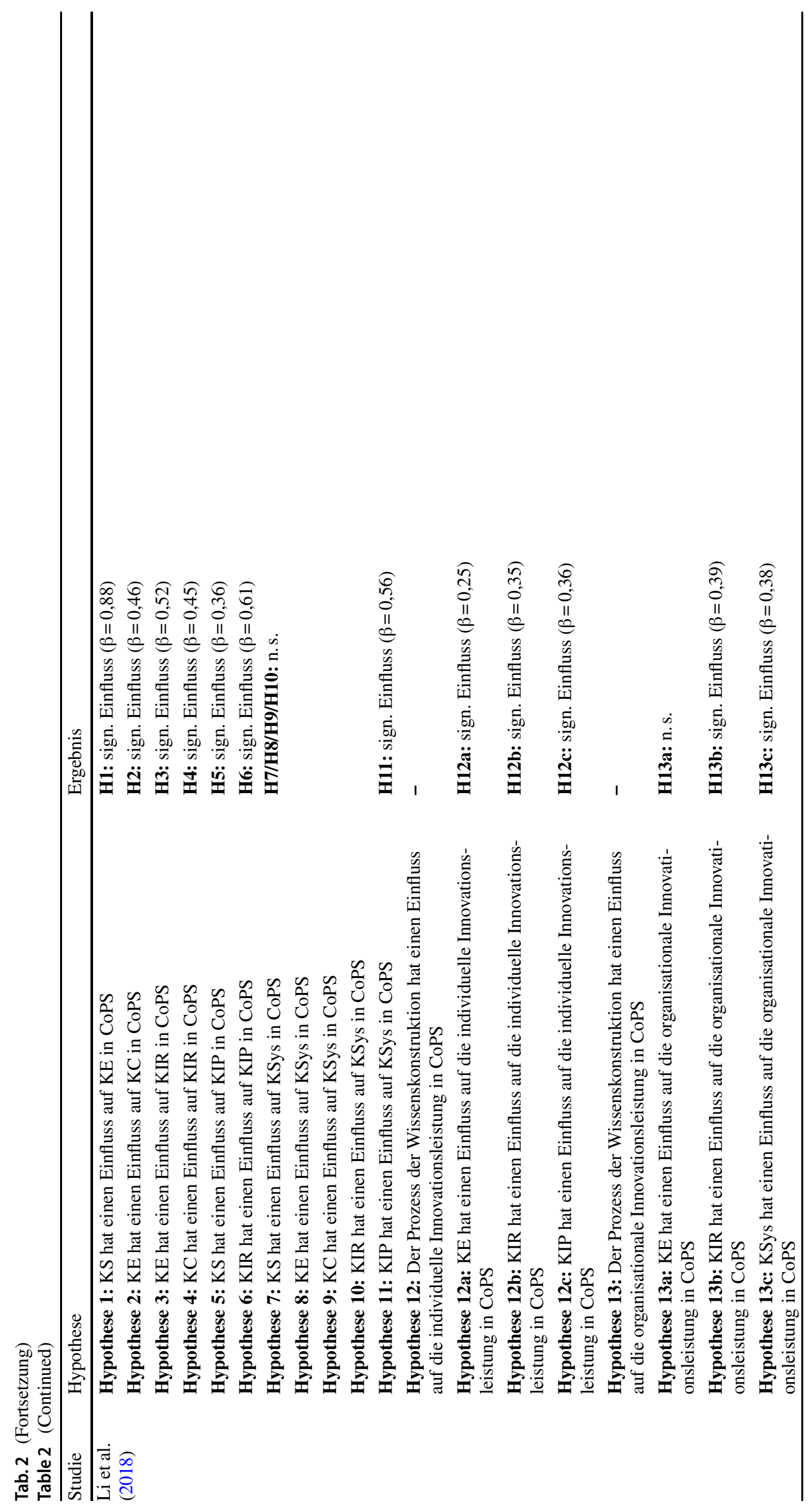


re die folgenden Wissenskonstruktionsprozesse: Wissenssozialisierung (KS), Wissensexternalisierung (KE), Wissenskombination (KC), Wissensinternalisierung durch Reflexion (KIR), Wissensinternalisierung durch Praxis (KIP) und Wissenssystematisierung (KSys). Zur Überprüfung der Zusammenhänge zwischen diesen Wissenskonstruktionsprozessen wurde eine Fragebogenerhebung durchgeführt, an der 169 Probandinnen und Probanden teilnahmen.

\subsection{Individuelles und kollektives Lernen}

Tab. 3 stellt die einbezogenen Artikel für die Betrachtung des Zusammenhangs zwischen individuellem und kollektivem Lernen zusammenfassend dar. Burmeister et al. (2018) gehen in ihrem Artikel von einem bidirektionalen Wissenstransfer aus, bei dem Wissen zwischen zwei Mitarbeitenden geteilt und aufgenommen wird. Studien konnten hier zeigen, dass die Rolle, die Mitarbeitenden innerhalb des Wissenstransfers übernehmen, vom Alter abhängt. So werden ältere Mitarbeitenden als die Sender von Wissen und jüngere Mitarbeitenden als Empfänger von Wissen betrachtet (Voelpel et al. 2012; Deal et al. 2010). Burmeister et al. (2018) greifen diese Befunde in ihrer Studie auf. Sie untersuchen zum einen den Einfluss des Alters auf die wahrgenommene Fähigkeit und die wahrgenommene Motivation, Wissen zu teilen sowie aufzunehmen; zum anderen die Glaubhaftigkeit als Moderatorvariable. Der Studie liegt ein experimentelles 2 (Alter: alt vs. jung; withinsubject) $\times 3$ (Glaubwürdigkeitsdimensionen: Kompetenz vs. Wohlwollen vs. Integrität, within-subject) $\times 2$ (Glaubwürdigkeitsniveau: niedrig vs. hoch, between-subject) gemischtes faktorielles Design zugrunde. In die Analysen wurden 379 Probandinnen und Probanden aufgenommen, die jeweils sechs Vignetten bewerten sollten.

Auch Gerpott et al. (2019) widmen sich dem Einfluss der Altersunterschiede auf das Teilen von Wissen. Die Fragestellung untersuchten Gerpott et al. (2019) über eine Fragebogenerhebung, die in eine Trainingsstudie eingebettet war, an der 211 Probandinnen und Probanden teilnahmen. Das Training verfolgte das Ziel, Prinzipien der kontinuierlichen Verbesserung zu vermitteln.

Kaše et al. (2019) untersuchten den Einfluss von Motivation auf den Prozess der Kompetenzentwicklung, bei dem Wissen von jüngeren Mentorinnen und Mentoren an ältere Lernende transferiert wird. Dazu nutzt die Autorenschaft eine Fragebogenerhebung in einer two-sample study, an der 457 Mentorinnen und Mentoren und 293 Lernende teilnahmen.

Han et al. (2020) widmen sich in ihrer Studie der Frage nach Einflussfaktoren auf das Teilen von Wissen zwischen Individuen. Die Untersuchung der Fragestellung wurden Netzwerkabfragen und Selbstberichte verwendet. Ins- gesamt wurde eine Netzwerkanalyse basierend auf 111 Teilnehmenden durchgeführt.

Auch Zhu et al. (2018) gehen in ihrer Studie dem Einfluss des Teilens von Wissen für die Sender und Empfänger des Wissens nach. In einer Fragebogenstudie wurden die Hypothesen an einer Stichprobe von 233 Probandinnen und Probanden getestet.

Ghosh und Tripathi (2020) untersuchten in ihrer Studie den Zusammenhang zwischen wahrgenommener Inklusion auf individueller als auch auf Gruppenebene und dem Team-Kreativitätsklima (TCC) sowie die Rolle des TeamLernklimas (TLC) und der Aufgaben-Interdependenz für diesen Zusammenhang. Zur Untersuchung der Fragestellung liegt der Studie eine Fragebogenerhebung zugrunde, an der 303 Probandinnen und Probanden teilnahmen.

Chan et al. (2021) untersuchen in ihrer Studie den Einfluss von multiple project team membership auf das individuelle Lernen sowie das Lernen in Gruppen. An der Fragebogenerhebung nahmen 455 Probandinnen und Probanden teil.

Lee (2019) ging in seiner Studie der Frage nach, inwiefern die räumliche Nähe zwischen Mitgliedern einer Organisation die Erkundungsbereitschaft auf individueller Ebene verbessert. Mithilfe eines natürlichen Experimentes wurde die Fragestellung untersucht, an dem 60 Probandinnen und Probanden über einen längeren Zeitraum teilnahmen, so dass insgesamt 38.435 Beobachtungen gemacht werden konnten.

Janardhanan et al. (2020) widmen sich der Frage, wie die Zielorientierung des Teams das Querverständnis beeinflusst, d.h. das Ausmaß, in dem die Teammitglieder die mentalen Modelle der anderen Mitglieder verstehen, was sich auf die Team- und die individuelle Leistung auswirkt. Der Studie liegt dabei eine Fragebogenerhebung zugrunde, an der 859 Probandinnen und Probanden teilnahmen.

Auch Rhee und Choi (2017) untersuchten in ihrer Studie den Einfluss der Zielorientierung auf das Wissensmanagement und den Prozess des Teilens von Wissen sowie auf die kreative Leistung des Individuums. Zur Untersuchung der Fragestellung wurde eine Fragebogenerhebung durchgeführt, an der 45 Managerinnen und Manager sowie 231 Teammitglieder teilnahmen. Dabei wurden alle Variablen in Form von Selbstberichtsbögen erfasst, außer die Einschätzung der Kreativität der Mitarbeitenden, die durch die Betreuer und Betreuerinnen erfolgten.

Zhang und Min (2019) untersuchten in ihrer Studie die negativen Folgen des Verbergens von Wissen auf die Gruppenleistung. Zur Untersuchung wurde eine Stichprobe von 92 Projektteams herangezogen, die an einer Fragebogenerhebung teilnahmen. Insgesamt umfasst die Studie eine Stichprobe von 92 Teamleitungen und 386 Teammitgliedern. 


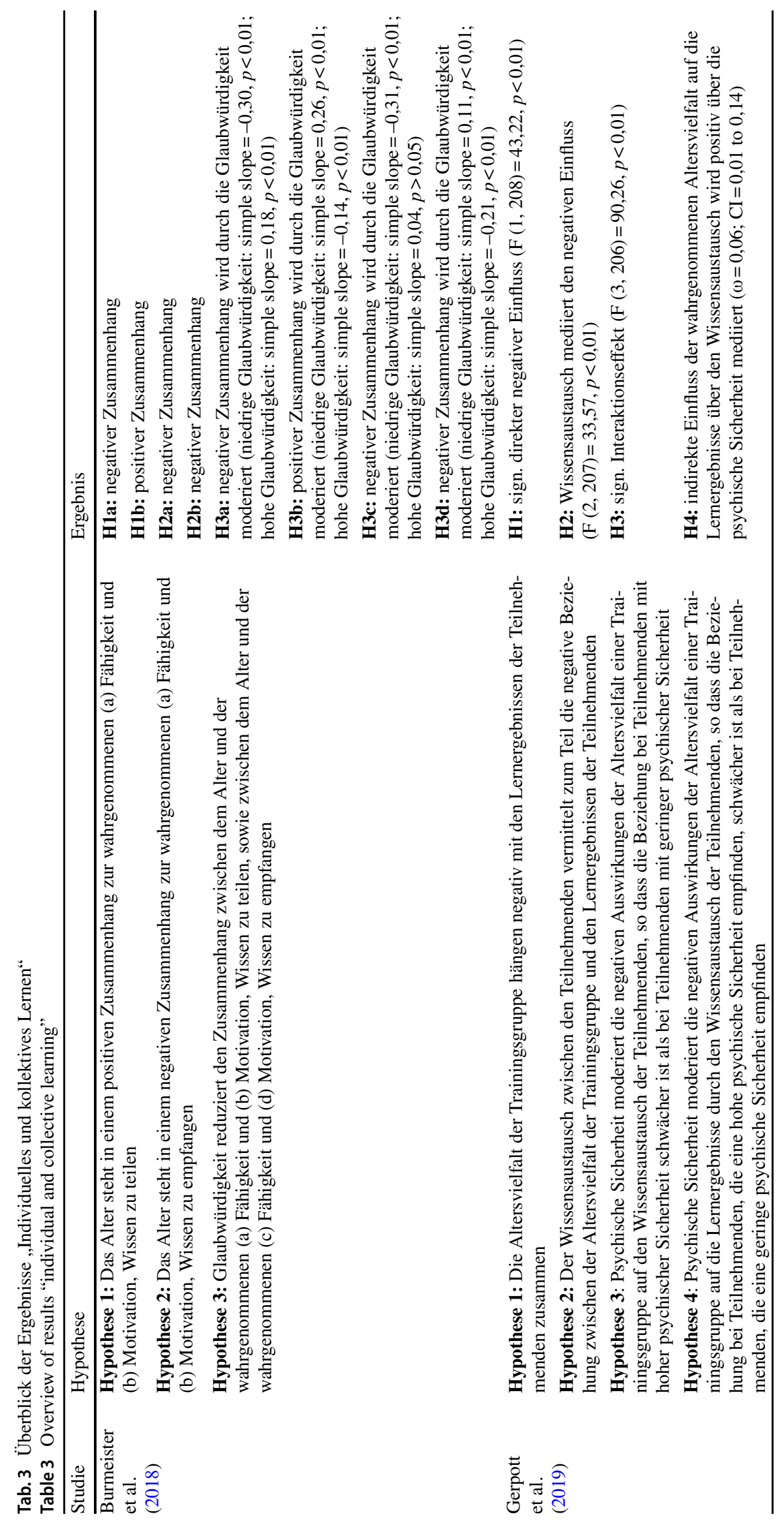




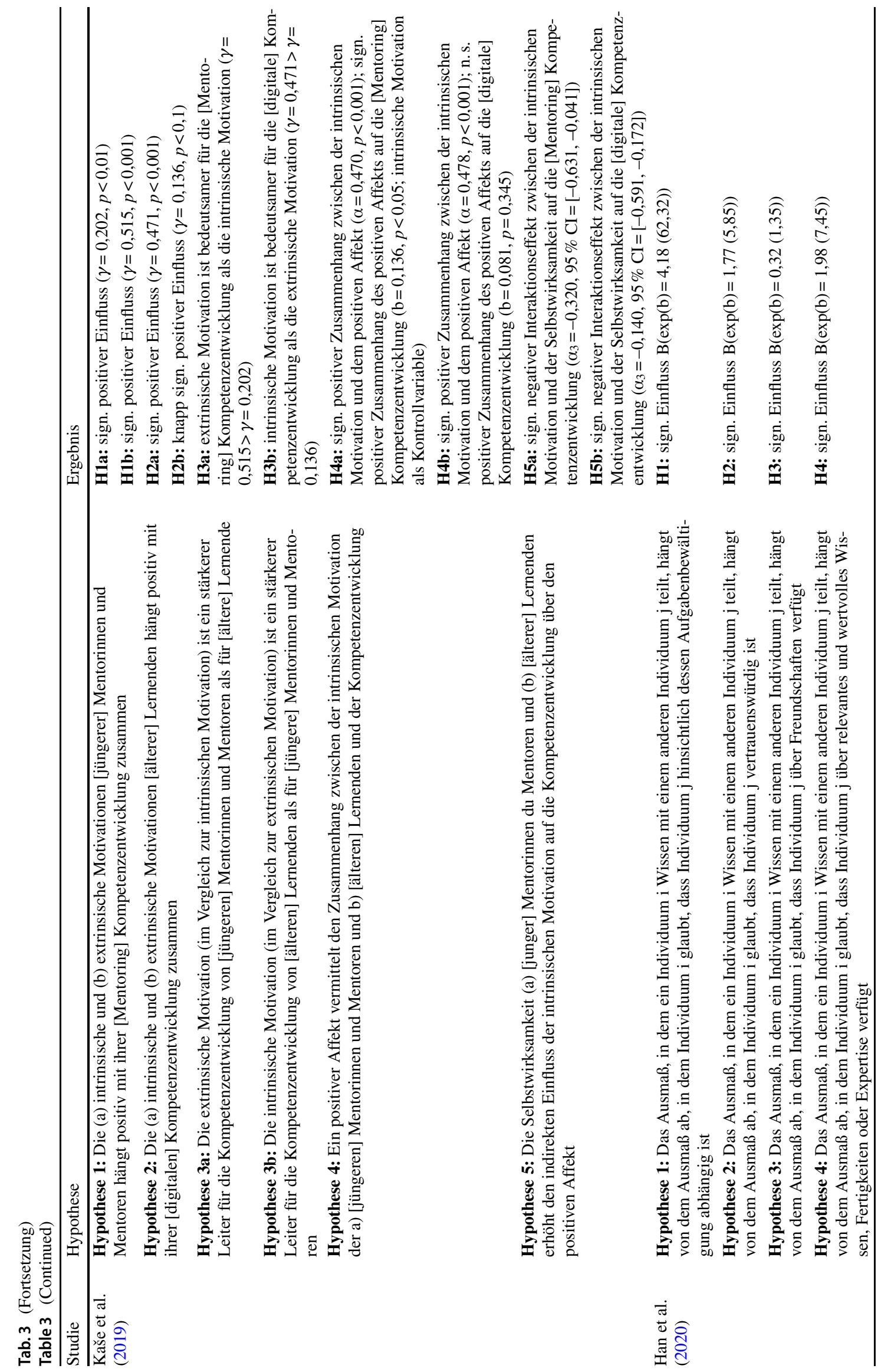




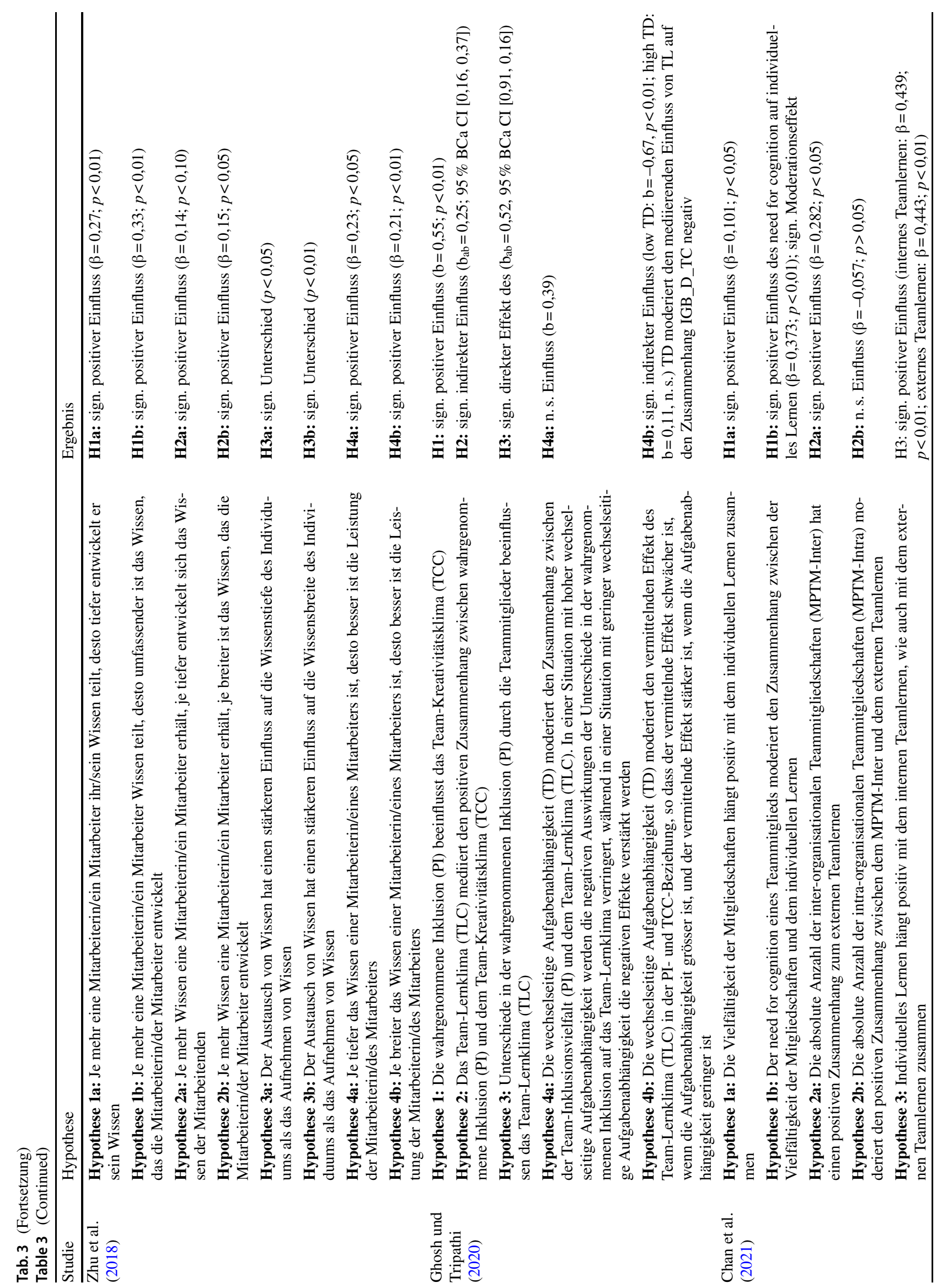




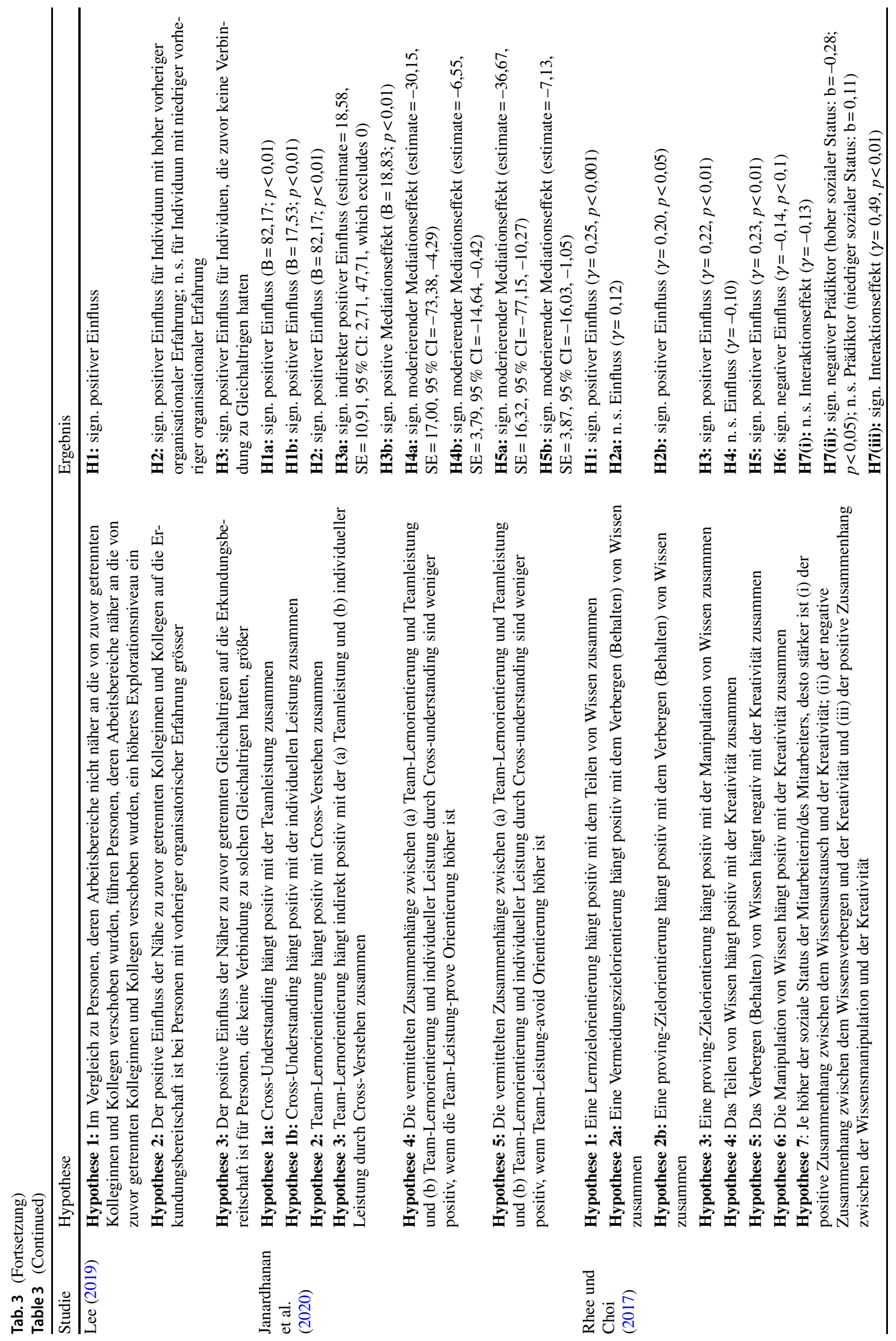




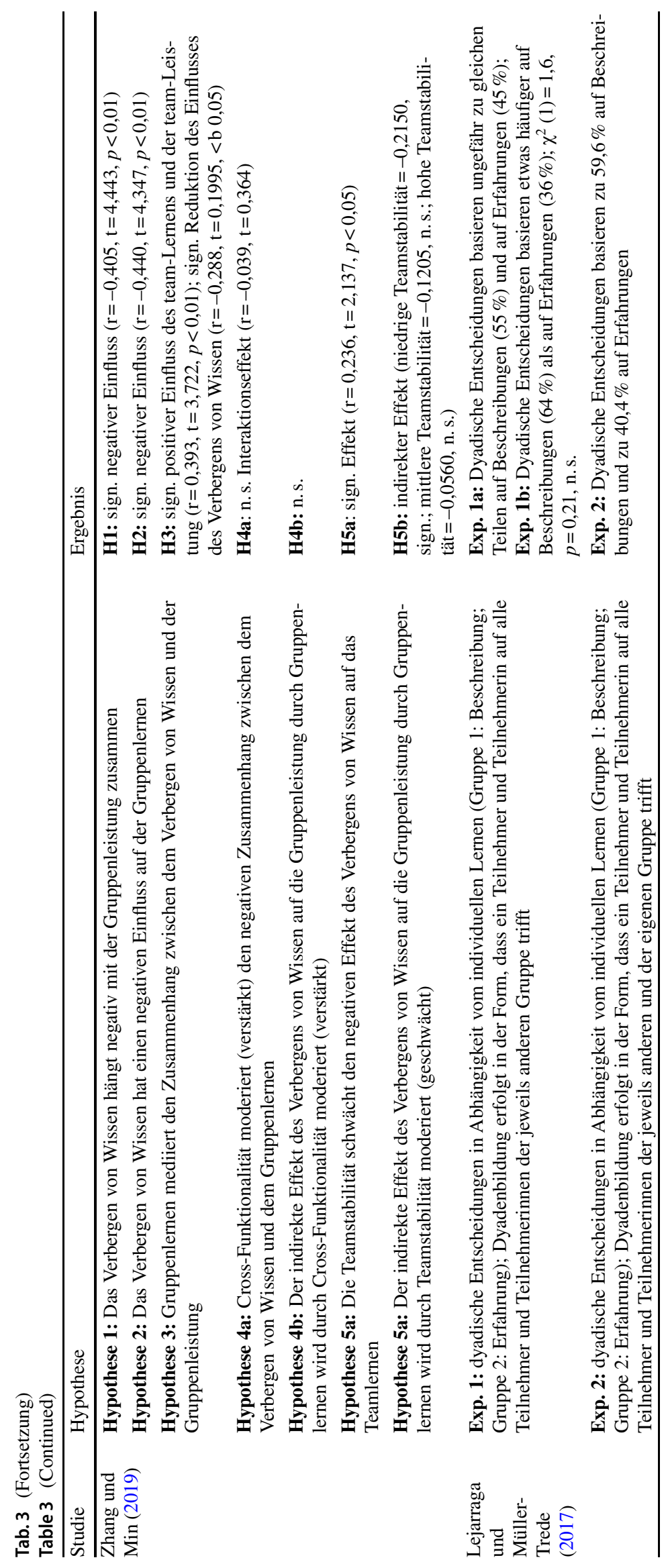


Lejarraga und Müller-Trede (2017) gehen in ihrer Studie der Frage nach, wie Gruppen gemeinsame Entscheidungen treffen, wenn die Individuen unterschiedlich lernen. Hierbei unterscheiden sie insbesondere zwischen dem Lernen durch beschreibende Informationen und Lernen durch Erfahrung. In einem experimentellen Design untersuchten die Autorenschaft, wie Partnergruppen Entscheidungen treffen, wenn das eine Gruppenmitglied durch beschreibende Informationen und das andere Gruppenmitglied aus Erfahrungen lernt. Für den vorliegenden Kontext ist insbesondere Experiment 1 von Relevanz, an dem insgesamt 156 Probandinnen und Probanden teilnahmen. Das Experiment bestand dabei aus einer individuellen Entscheidungsfindung und einer dyadischen Entscheidungsfindung. Die Analysen aus Experiment 1 zeigen, dass sich die Entscheidungen der Partnergruppen ungefähr genauso wahrscheinlich an den Entscheidungen des Mitglieds orientieren, das aus der Beschreibung lernt, wie auch an den Entscheidungen des Mitglieds, das aus Erfahrung lernt. Dyadische Entscheidungen reflektieren somit einen gemeinsamen Konsens.

\section{Diskussion und Ausblick}

Das vorliegende Literatur-Review zeigt, dass Lernen in Organisationen zum einen auf dem Zusammenspiel der drei Akteursebenen (Individuum, Gruppe, Organisation) gedacht werden muss und zum anderen sowohl individuelle, gruppenbasierte als auch organisationale Faktoren einen Einfluss auf das Lernen haben. Ein Schwerpunkt, der sich anhand des Literatur-Reviews herausarbeiten lässt, ist die Relevanz des Wissensaustauschs bzw. des Teilens von Wissen. So zeigt z.B. die Studie von Zhang und Min (2019), dass das Verbergen von Wissen, d.h. ein fehlender Wissensaustausch zwischen Individuen, einen negativen Einfluss auf die Gruppenleistung hat. Auch die Studie von Zhu et al. (2018) betont die Relevanz des Wissensaustauschs für alle Beteiligten dahingehend, als ein erhöhter Wissensaustausch sowohl beim Sender als auch beim Empfänger zu einer tieferen und breiteren Wissensentwicklung beiträgt. Berücksichtigt man die Relevanz des Wissensaustauschs für den Lernerfolg innerhalb einer Gruppe, so kann angenommen werden, dass ein Wissensaustausch auch zwischen Gruppen einen positiven Einfluss auf das Lernen auf organisationaler Ebene hat. Dies unterstützen die Erkenntnisse von Akinci und Sadler-Smith (2019) auf das Lernen auf der Gruppenebene als Bindeglied.

Die im Rahmen des Literatur-Reviews aufgeführten Studien gehen dabei der Identifikation von Faktoren nach, die den Wissensaustausch zwischen Individuen positiv beeinflussen. Hierzu zählen:
- individuelle Faktoren, wie z.B. das Alter, die Zielorientierung, die Flexibilität der Mitarbeitenden, das psychologische Kapital, die psychische Sicherheit, Vielfältigkeit der Projektmitgliedschaften oder die Motivation

- gruppenbasierte Faktoren, wie z.B. die Wahrnehmung der Teammitglieder (Glaubwürdigkeit), Altersvielfalt, Teamstabilität, der soziale Status innerhalb der Gruppe oder das Zugehörigkeitsgefühl

- organisationale Faktoren, wie z.B. organisationale Lernfähigkeit, die Person-Organisations-Passung, die PersonJob-Passung oder Prozesse des Wissensmanagements

- Rahmenbedingungen, wie z.B. die räumliche Nähe zwischen Individuen

Insgesamt zeigt das Literatur-Review auf, dass vielfältige Faktoren den Wissensaustausch begünstigen können. Für Organisationen ist es somit von großer Relevanz diese Faktoren zu berücksichtigen, um organisationales Lernen wirksam auf allen Akteursebenen zu gestalten. Organisationale Veränderungsprozesse können und müssen als kollaborative Aufgabe wahrgenommen werden, zu der jedes Individuum einen Beitrag leisten kann. Zukünftige Forschung sollte die aufgeführten Faktoren näher berücksichtigen und Entwicklungsmöglichkeiten identifizieren und hinsichtlich ihrer Wirksamkeit evaluieren, um die Faktoren mit negativem Einfluss zu entkräften und Faktoren mit positivem Einfluss zu stärken. So wird kann z.B. anhand des Einflussfaktors der Teamstabilität oder dem Zugehörigkeitsgefühl angenommen werden, dass Team-Meetings, insbesondere informelle, an Relevanz für organisationales Lernen gewinnen, da hier das Team gestärkt wird. Fühlt sich ein Individuum alleine, trägt dies dazu bei, dass Wissen nicht geteilt, sondern vor anderen geschützt wird. Ziel jeder Organisation muss es sein, Gelegenheiten zu schaffen, in denen Teamentwicklungs-Maßnahmen genutzt werden können, um den Weg vom Einzelkämpferdenken zum kollaborativen Teamdenken und einem Gefühl der Gemeinschaft ebnen zu können, um organisationales Lernen zu fördern. Auch in Zeiten der Digitalisierung, in denen digitale Lernformen, wie z.B. das Remote Learning an Bedeutung gewinnen, sollten informelle Kommunikationsgelegenheiten und die Nähe zu anderen Mitarbeitenden nicht vernachlässigt werden, da sie essentiell zum organisationalen Lernerfolg beitragen.

Funding Open Access funding enabled and organized by Projekt DEAL.

Open Access Dieser Artikel wird unter der Creative Commons Namensnennung 4.0 International Lizenz veröffentlicht, welche die Nutzung, Vervielfältigung, Bearbeitung, Verbreitung und Wiedergabe in jeglichem Medium und Format erlaubt, sofern Sie den/die ursprünglichen Autor(en) und die Quelle ordnungsgemäß nennen, einen Link zur Creative Commons Lizenz beifügen und angeben, ob Änderungen vorgenommen wurden. 
Die in diesem Artikel enthaltenen Bilder und sonstiges Drittmaterial unterliegen ebenfalls der genannten Creative Commons Lizenz, sofern sich aus der Abbildungslegende nichts anderes ergibt. Sofern das betreffende Material nicht unter der genannten Creative Commons Lizenz steht und die betreffende Handlung nicht nach gesetzlichen Vorschriften erlaubt ist, ist für die oben aufgeführten Weiterverwendungen des Materials die Einwilligung des jeweiligen Rechteinhabers einzuholen.

Weitere Details zur Lizenz entnehmen Sie bitte der Lizenzinformation auf http://creativecommons.org/licenses/by/4.0/deed.de.

\section{Literatur}

Adams P (2006) Exploring social constructivism: theories and practicalities. Education 3-13 34(3):243-257 https://doi.org/10.1080/ 03004270600898893

Akinci C, Sadler-Smith E (2019) Collective Intuition: Implications for Improved Decision Making and Organizational Learning. Brit J Manage 30(3):558-577

Annosi MC, Martini A, Brunetta F, Marchegiani L (2018) Learning in an agile setting: A multilevel research study on the evolution of organizational routines. J Bus Res 110:554-566

Aponte SPD, Zapata DIC (2013) A model of organizational learning in practice. Estudios Gerenciales 29(129):439-444

Argote L, Levine JM (Hrsg) (2020) The Oxford handbook of group and organizational learning. Oxford Library of Psychology. Oxford University Press, New York

Argyris C, Schön DA (1978) Organizational learning. Organization development deries. Addison-Wesley Pub, Co, Reading, Mass

Argyris C, Schön DA (1996) Organizational learning II: Theory, method and practice. Organization development series. AddisonWesley, Reading, Mass, Wokingham

Bain A (1998) Social Defenses Against Organizational Learning. Hum Relations 51(3):413-429

Bandura A (1988) Organisational Applications of Social Cognitive Theory. Aust J Manag 13(2):275-302

Bennett, Joan KremerlO'Brien, Michael J. (1994) The Building Blocks of the Learning Organization. Training 31(6):41

Berg D (1993) Expanding perceptions, possibilities and profits. J Qual Particip 16(7):6-10

Bierly PE, Hämäläinen T (1995) Organizational learning and strategy. Scand J Manag 11(3):209-224

Brown JS, Duguid P (1998) Organizing Knowledge. Calif Manage Rev 40(3):90-111

Burmeister A, Fasbender U, Deller J (2018) Being perceived as a knowledge sender or knowledge receiver: A multistudy investigation of the effect of age on knowledge transfer. J Occup Organ Psychol 91(3):518-545

Camps J, Oltra V, Aldás-Manzano J, Buenaventura-Vera G, TorresCarballo F (2016) Individual Performance in Turbulent Environments: The Role of Organizational Learning Capability and Employee Flexibility. Hum Resour Manage 55(3):363-383

Cerasoli CP, Alliger GM, Donsbach JS, Mathieu JE, Tannenbaum SI, Orvis KA (2018) Antecedents and Outcomes of Informal Learning Behaviors: a Meta-Analysis. J Bus Psychol 33(2):203-230

Chan CCA (2003) Examining the relationships between individual, team and organizational learning in an Australian hospital. Learn Health Soc Care 2(4):223-235

Chan K-Y, Oerlemans L, Meslec N (2021) The impact of multiple project team membership on individual and team learning: A micromeso multi-level empirical study. Int J Proj Manag 39(3):308-320

Choi W, Noe R, Cho Y (2020) What is responsible for the psychological capital-job performance relationship? An examination of the role of informal learning and person-environment fit. JMP $35(1): 28-41$
Chonko LB, Dubinsky AJ, Jones E, Roberts JA (2003) Organizational and individual learning in the sales force: an agenda for sales research. J Bus Res 56(12):935-946

Crossan MM, Lane HW, White RE (1999) An Organizational Learning Framework: From Intuition to Institution. AMR 24(3):522-537. https://doi.org/10.5465/amr.1999.2202135

Deal JJ, Altman DG, Rogelberg SG (2010) Millennials at Work: What We Know and What We Need to Do (If Anything). J Bus Psychol 25(2):191-199

Dechant K, Marsick V, Kasl E (2000) Team learning: a model for effectiveness in high performing teams. In: Beyerlein IM, Johnson DA, Beyerlein ST (Hrsg) Team Development. Elsevier, New York, NY.

Desai VM (2020) Can Busy Organizations Learn to Get Better? Distinguishing Between the Competing Effects of Constrained Capacity on the Organizational Learning Process. Organ Sci 31(1):67-84

Endruweit G (2004) Organisationssoziologie. Lucius \& Lucius, Stuttgart

Furlan A, Galeazzo A, Paggiaro A (2019) Organizational and perceived learning in the workplace: A multilevel perspective on employees' problem solving. Organ Sci 30(2):280-297

Garratt B (1990) Creating a Learning Organization: a Guide to Leadership, Learning and Development. In: Lafferty, K. (1991). Creating a Learning Organisation: A Guide to Leadership, Learning and Development. Cambridge: Director Books.

Gattermeyer W, Al-Ani A (2001) Von individueller Veränderung zu organisationaler Transformation. Eine Einleitung. In: Change Management und Unternehmenserfolg. Gabler, S 7-10

Geng S, Chuah KB, Law KMY, Cheung CK, Chau YC, Rui C (2018) Knowledge Contribution as a Factor in Project Selection. Proj Manag J 49(1):25-41

Gerpott FH, Lehmann-Willenbrock N, Wenzel R, Voelpel SC (2019) Age diversity and learning outcomes in organizational training groups: the role of knowledge sharing and psychological safety. Int J Hum Resour Manag. https://doi.org/10.1080/09585192. 2019.1640763

Ghosh V, Tripathi N (2020) Perceived inclusion and team creativity climate: examining the role of learning climate and task interdependency. Manag Res Rev 44(6):849-866

Greco LM, Charlier SD, Brown KG (2019) Trading off learning and performance: Exploration and exploitation at work. Hum Resour Manag Rev 29(2):179-195

Greve HR, Rudi N, Walvekar A (2019) Rational Fouls? Loss aversion on organizational and individual goals influence decision quality. Organ Stud 42(7):1031-1051

Han SH, Yoon SW, Chae C (2020) Building social capital and learning relationships through knowledge sharing: a social network approach of management students' cases. J Knowl Manag 24(4):921-939

Hayes J, Allinson CW (1998) Cognitive Style and the Theory and Practice of Individual and Collective Learning in Organizations. Hum Relations 51(7):847-871

Hogarth RM (2001) Educating intuition. University of Chicago Press, Chicago

Janardhanan NS, Lewis K, Reger RK, Stevens CK (2020) Getting to know you: Motivating cross-understanding for improved team and individual performance. Organ Sci 31(1):103-118

Kaše R, Saksida T, Mihelič KK (2019) Skill development in reverse mentoring: Motivational processes of mentors and learners. Hum Resour Manage 58(1):57-69

Kim DH (1993) A framework and methodology for linking individual and organizational learning: Application in TQM and Product Development. Dissertation, Massachusetts Institute of Technology

King WR (2001) Strategies for Creating A Learning Organization. Inf Syst Manag 18(1): 12-20

Klimecki R, Thomae M (1997) Organisationales Lernen : eine Bestandsaufnahme der Forschung. Management Forschung und Praxis (18) 
Kolb DA (1984) Experiential learning: Experience as the source of learning and development. Prentice-Hall, Englewood Cliffs, N.J

Lee S (2019) Learning-by-moving: Can reconfiguring spatial proximity between organizational members promote individuallevel exploration? Organ Sci 30(3):467-488

Lefrancois GR, Leppmann PK, Angermeier WF, Thiekötter TJ (1986) Psychologie des Lernens. Springer, Lehrbuch. Springer, Berlin, Heidelberg

Lejarraga T, Müller-Trede J (2017) When experience meets description: How dyads integrate experiential and descriptive information in risky decisions. Manage Sci 63(6):1953-1971

Levitt B, March JG (1988) Organizational Learning. Annu Rev Sociol 14(1):319-338. https://doi.org/10.1146/annurev.so.14.080188. 001535

Li M, Liu H, Zhou J (2018) G-SECI model-based knowledge creation for CoPS innovation: the role of grey knowledge. J Knowl Manag 22(4):887-911

Mainert J, Niepel C, Lans T, Greiff S (2018) How employees perceive organizational learning: construct validation of the 25 -item short form of the strategic learning assessment map (SF-SLAM). J Knowl Manag 22(1):57-75

March JG, Simon HA (1976) Kognitive grenzen der rationalität. In: March JG, Simon HA (Hrsg) Organisation und Individuum. Gabler Verlag, Wiesbaden, S 129-159

McLeod G (2003) Learning theory and instructional design

Mohrman SA, Cohen SG, Mohrman AM (1995) Designing team-based organizations: New forms for knowledge work. The. Jossey-Bass, management series. Jossey-Bass, San Francisco

Rhee YW, Choi JN (2017) Knowledge management behavior and individual creativity: Goal orientations as antecedents and ingroup social status as moderating contingency. J Organiz Behav 38(6):813-832
Senge PM (1990) The fifth discipline: The art and practice of the learning organization. Doubleday/Currency, New York, N.Y., London

Sorrells-Jones J (1999) The role of the chief nurse executive in the knowledge-intense organization of the future. Nurs Adm Q 23(3): $17-25$

Valentine MA (2017) Renegotiating Spheres of Obligation: The Role of Hierarchy in Organizational Learning. Adm Sci Q 63(3):570-606

Vera D, Crossan M, Apaydin M (2012) A Framework for Integrating Organizational Learning, Knowledge, Capabilities, and Absorptive Capacity. In: Easterby-Smith M, Lyles MA (Hrsg) Handbook of Organizational Learning and Knowledge Management. John Wiley \& Sons, Inc, Hoboken, NJ, USA, S 153-180

Voelpel S, Sauer A, Biemann T (2012) Career Planning for Mid- and Late-Career Workers. Oxford University Press,

Vygotsky LS (1975) Thought and language. MIT Press, Cambridge, Mass

Wang CL, Ahmed PK (2002) A review of the concept of organisational learning. Wolverhampton: University of Wolverhampton, Wolverhampton

Yates JF, Tschirhart MD (2006) Decision-making expertise. In: Ericsson KA, Charness N, Feltovich PJ, Hoffman RR (Hrsg) The Cambridge Handbook of Expertise and Expert Performance. Cambridge University Press, New York, NY, S 421-438

Zhang Z, Min M (2019) The negative consequences of knowledge hiding in NPD project teams: The roles of project work attributes. Int J Proj Manag 37(2):225-238

Zhu Q, Krikke H, Caniëls MCJ (2018) Supply chain integration: value creation through managing inter-organizational learning. Int $\mathrm{J}$ Oper Prod Manag 38(1):211-229 